\title{
UNICITY THEOREMS FOR MEROMORPHIC FUNCTIONS SHARING FOUR SMALL FUNCTIONS
}

\author{
Katsuya Ishizaki and Nobushige Toda
}

\begin{abstract}
Let $f$ and $g$ be transcendental meromorphic functions in the complex plane having properties $\bar{N}(r, f) \leq u T(r, f)+S(r, f)$ and $\bar{N}(r, g) \leq v T(r, g)+S(r, g)$ for some constants $u$ and $v$ satısfying $(u, v) \in[0,1 / 19) \times[0,1 / 19)$. If there exist four distinct small meromorphic functions shared by $f$ and $g$, then $f=g$.
\end{abstract}

\section{Introduction}

Let $f(z)$ be a transcendental meromorphic function in the complex plane and let $\boldsymbol{S}(f)$ be the set of meromorphic functions $a(z)$ in the complex plane which satisfy

$$
T(r, a)=S(r, f),
$$

where $S(r, f)$ is any quantity satisfying

$$
S(r, f)=o(T(r, f))
$$

for $r \rightarrow \infty$ except possibly a set of $r$ of finite linear measure. Such a meromorphic function $a(z)$ is said to be small with respect to $f$. Note that $\boldsymbol{S}(f)$ is a field.

We put for $a \in \boldsymbol{S}(f) \cup\{\infty\}$

$$
E(f=a)=\{z: f(z)-a(z)=0\},
$$

where $f(z)-\infty$ means $1 / f(z)$.

Throughout the paper we shall use the standard notation of the Nevanlinna theory of meromorphic functions ([4], [5]) and the followings.

For a positive integer $k$ let

$$
n_{k)}\left(r, \frac{1}{f}\right)\left(\text { resp. } n_{(k}\left(r, \frac{1}{f}\right)\right)
$$

1991 Mathematics Subject Classification. 30D35.

Key words and phrases. Unicity theory, Meromorphic functions, Small functions.

This work was supported in part by a Grant-1n-A1d for General Scientific Research from the Ministry of Education, Science and Culture 09640180 and 09740118.

Received May 8, 1998. 
be the number of zeros of $f$ with order $\leq k$ (resp. $\geq k$ ) counting multiplicities in $|z| \leq r$ and put for $r>0$

$$
N_{k)}\left(r, \frac{1}{f}\right)=\int_{0}^{r} \frac{n_{k)}\left(t, \frac{1}{f}\right)-n_{k)}\left(0, \frac{1}{f}\right)}{t} d t+n_{k)}\left(0, \frac{1}{f}\right) \log r
$$

(resp.

$$
\left.N_{(k}\left(r, \frac{1}{f}\right)=\int_{0}^{r} \frac{n_{(k}\left(t, \frac{1}{f}\right)-n_{(k}\left(0, \frac{1}{f}\right)}{t} d t+n_{(k}\left(0, \frac{1}{f}\right) \log r\right) .
$$

Similarly for a positive integer $k$ let

$$
\bar{n}_{k)}\left(r, \frac{1}{f}\right)\left(\text { resp. } \bar{n}_{(k}\left(r, \frac{1}{f}\right)\right)
$$

be the number of zeros of $f$ with order $\leq k$ (resp. $\geq k$ ) ignoring multiplicities in $|z| \leq r$ and put for $r>0$

$$
\bar{N}_{k)}\left(r, \frac{1}{f}\right)=\int_{0}^{r} \frac{\bar{n}_{k)}\left(t, \frac{1}{f}\right)-\bar{n}_{k)}\left(0, \frac{1}{f}\right)}{t} d t+\bar{n}_{k)}\left(0, \frac{1}{f}\right) \log r
$$

(resp.

$$
\left.\bar{N}_{(k}\left(r, \frac{1}{f}\right)=\int_{0}^{r} \frac{\bar{n}_{(k}\left(t, \frac{1}{f}\right)-\bar{n}_{(k}\left(0, \frac{1}{f}\right)}{t} d t+\bar{n}_{(k}\left(0, \frac{1}{f}\right) \log r\right) .
$$

The truncated counting function $N_{k}(r, 1 / f)$ is defined by

$$
N_{k}\left(r, \frac{1}{f}\right)=N_{k)}\left(r, \frac{1}{f}\right)+k \bar{N}_{(k+1}\left(r, \frac{1}{f}\right) .
$$

Then, $\bar{N}(r, 1 / f)=N_{1}(r, 1 / f)$.

Let $f$ and $g$ be two transcendental meromorphic functions in the complex plane. The following "Unicity Theorem" is well-known:

Unicity TheOREm of Nevanlinna. If for five distinct elements $a_{1}, \ldots, a_{5} \in$ $C \cup\{\infty\}$

$$
E\left(f=a_{j}\right)=E\left(g=a_{j}\right) \quad(j=1, \ldots, 5),
$$

then $f=g([5]$, p. 109; see also [4], p. 48).

This theorem is proved by making use of Lemma 1(a) given in Section 2. This theorem is sharp ([5], p. 111). It is quite natural to ask whether it is possible to extend this theorem to the case when $a_{1}, \ldots, a_{5}$ belong to $\{\boldsymbol{S}(f) \cap \boldsymbol{S}(g)\} \cup\{\infty\}$ (see [1], [6]). Since there is no result similar to Lemma 1(a) for $a_{1}, \ldots, a_{q} \in \boldsymbol{S}(f) \cup\{\infty\}([2])$, this problem is not easy to solve. 
Several results on this problem are known (see [7], [8]). For example, for $a, b \in \boldsymbol{S}(f)$ such that $0,1, a, b$ are distinct and one of $a$ and $b$ is not constant, Q.D. Zhang ([8]) used the determinant

$$
\left|\begin{array}{ccc}
f f^{\prime} & f^{\prime} & f^{2}-f \\
a a^{\prime} & a^{\prime} & a^{2}-a \\
b b^{\prime} & b^{\prime} & b^{2}-b
\end{array}\right|
$$

to prove the following

THEOREM A. Let $f$ and $g$ be two transcendental meromorphic functions in the complex plane. If for six distinct elements $a_{1}, \ldots, a_{6} \in\{\boldsymbol{S}(f) \cap \boldsymbol{S}(g)\} \cup\{\infty\}$

$$
E\left(f=a_{j}\right)=E\left(g=a_{j}\right) \quad(j=1, \ldots, 6),
$$

then, $f=g$ (Theorem 2 in [8]).

It is said that $f$ and $g$ share $a$ if $E(f=a)=E(g=a)$ for a meromorphic function $a$ in the complex plane.

The purpose of this paper is to consider the unicity theorem from a slightly different point of view. We shall give some unicity theorems for transcendental meromorphic functions sharing four distinct small meromorphic functions and satisfying some conditions on the counting functions of poles. For example, the following proposition is a special case of Corollary 1 .

Proposition. Let $f$ and $g$ be transcendental meromorphic functions in the complex plane satisfying

$$
\bar{N}(r, f) \leq u T(r, f)+S(r, f) \quad \text { and } \quad \bar{N}(r, g) \leq u T(r, g)+S(r, g)
$$

for a constant $u$ in $[0,1 / 19)$.

If there exist four distinct functions $a_{1}, a_{2}, a_{3}, a_{4}$ in $\boldsymbol{S}(f) \cap \boldsymbol{S}(g)$ such that

$$
E\left(f=a_{j}\right)=E\left(g=a_{j}\right) \quad(j=1,2,3,4)
$$

then $f=g$.

Note that $f$ and $g$ do not always share $\infty$.

As a corollary, we shall give a unicity theorem for meromorphic functions sharing five distinct small meromorphic functions.

\section{Preliminary and lemma}

(I) We shall give some lemmas first.

LEMMA 1. Let $f(z)$ be a transcendental meromorphic function in the complex plane. Then we have the following inequalities: 
(a) For $q$ elements $a_{1}, \ldots, a_{q} \in C \cup\{\infty\}(q<\infty)$,

$$
(q-2) T(r, f) \leq \sum_{j=1}^{q} \bar{N}\left(r, \frac{1}{f-a_{j}}\right)+S(r, f) .
$$

(b) For three distinct elements $a_{1}, a_{2}$ and $a_{3} \in \boldsymbol{S}(f) \cup\{\infty\}$

$$
T(r, f) \leq \sum_{j=1}^{3} \bar{N}\left(r, \frac{1}{f-a_{j}}\right)+S(r, f) .
$$

(c) For five distinct elements $a_{1}, \ldots, a_{5} \in \boldsymbol{S}(f) \cup\{\infty\}$

$$
3 T(r, f) \leq \sum_{i=1}^{5} \bar{N}\left(r, \frac{1}{f-a_{i}}\right)+2\left\{\bar{N}\left(r, \frac{1}{f-a_{j}}\right)+\bar{N}\left(r, \frac{1}{f-a_{k}}\right)\right\}+S(r, f)
$$

for any $j, k(1 \leq j \neq k \leq 5)$.

Proof of Lemma 1. (a) and (b) are famous inequalities given in [5]. We can easily obtain (c) from (b). In fact, for any fixed $1 \leq j \neq k \leq 5$ we apply (b) to $a_{i}, a_{j}, a_{k}(1 \leq i \leq 5, i \neq j, k)$ to obtain the following three inequalities

$$
\begin{aligned}
T(r, f) \leq & \bar{N}\left(r, \frac{1}{f-a_{i}}\right)+\bar{N}\left(r, \frac{1}{f-a_{j}}\right) \\
& +\bar{N}\left(r, \frac{1}{f-a_{k}}\right)+S(r, f)(1 \leq i \leq 5, i \neq j, k) .
\end{aligned}
$$

By adding them side by side we obtain (c).

LEMMA 2 ([8]). Let $f(z)$ be a transcendental meromorphic function in the complex plane and let $a_{1}, \ldots, a_{5}$ be five distinct elements of $\boldsymbol{S}(f) \cup\{\infty\}$. We then have the following inequality:

$$
2 T(r, f) \leq \sum_{j=1}^{5} \bar{N}\left(r, \frac{1}{f-a_{j}}\right)+S(r, f)
$$

LEMMA 3 ([3]). Let $f(z)$ be a transçendental meromorphic function in $|z|<\infty$. Then for any $q$ distinct elements $a_{1}, \ldots, a_{q} \in \boldsymbol{S}(f)(2 \leq q \leq \infty)$ the following inequality holds:

$$
(q-1) T(r, f) \leq \sum_{j=1}^{q} N_{m}\left(r, \frac{1}{f-a_{j}}\right)+m \bar{N}(r, f)+S(r, f),
$$

where $m$ is the number of elements of a maximal linearly independent subset of $\left\{a_{1}, \ldots, a_{q}\right\}$. 
Proof of Lemma 3. This is essentially contained in the proof of Theorem in [3]. Let $\left\{b_{1}, \ldots, b_{m}\right\}$ be a maximal linearly independent subset of $\left\{a_{1}, \ldots, a_{q}\right\}$ and put

$$
L(f)=W\left(b_{1}, \ldots, b_{m}, f\right),
$$

where $W\left(b_{1}, \ldots, b_{m}, f\right)$ is the Wronskian of $b_{1}, \ldots, b_{m}, f$. Then $L(f) \neq 0$ as $b_{1}, \ldots, b_{m}$ and $f$ are linearly independent. The relation ([3], pp. 80-81)

$$
\sum_{j=1}^{q} m\left(r, \frac{1}{f-a_{j}}\right)=m\left(r, \sum_{j=1}^{q} \frac{A_{j}}{f-a_{j}}\right)+S(r, f),
$$

where $A_{j}^{\prime}$ s are small with respect to $f$, and the inequality ([3], p. 81)

$$
m\left(r, \sum_{j=1}^{q} \frac{A_{j}}{f-a_{j}}\right) \leq T(r, f)+m \bar{N}(r, f)-N\left(r, \frac{1}{L(f)}\right)+S(r, f)
$$

hold. Combining these relations we obtain the inequality

$$
\sum_{j=1}^{q} m\left(r, \frac{1}{f-a_{j}}\right) \leq T(r, f)+m \bar{N}(r, f)-N\left(r, \frac{1}{L(f)}\right)+S(r, f) .
$$

We add $\sum_{J=1}^{q} N\left(r, 1 /\left(f-a_{j}\right)\right)$ to both sides of this inequality, then due to the first fundamental theorem of Nevanlinna we obtain the inequality

$$
\begin{aligned}
(q-1) T(r, f) & \leq \sum_{j=1}^{q} N\left(r, \frac{1}{f-a_{j}}\right)-N\left(r, \frac{1}{L(f)}\right)+m \bar{N}(r, f)+S(r, f) \\
& \leq \sum_{j=1}^{q} N_{m}\left(r, \frac{1}{f-a_{j}}\right)+m \bar{N}(r, f)+S(r, f)
\end{aligned}
$$

since

$$
\sum_{j=1}^{q} N\left(r, \frac{1}{f-a_{j}}\right)-N\left(r, \frac{1}{L(f)}\right) \leq \sum_{j=1}^{q} N_{m}\left(r, \frac{1}{f-a_{j}}\right)+S(r, f) .
$$

LEMMA 4. Let $f$ and $g$ be nonconstant meromorphic functions in $|z|<\infty$ satisfying

$$
\frac{\left(f^{\prime}\right)^{2}}{f(f-1)}=\frac{\left(g^{\prime}\right)^{2}}{g(g-1)}
$$

Then, $T(r, g)=T(r, f)+O(1)$.

Proof of Lemma 4. Put $F=2 f-1$ and $G=2 g-1$. Then from (1)

$$
\frac{\left(F^{\prime}\right)^{2}}{F^{2}-1}=\frac{\left(G^{\prime}\right)^{2}}{G^{2}-1}
$$


We denote by $A$ the common function in (2). Then, we have

$$
\frac{\left(F^{\prime}\right)^{2}}{A}=F^{2}-1 \text { and } \quad \frac{\left(G^{\prime}\right)^{2}}{A}=G^{2}-1 .
$$

Differentiate them to obtain

$$
\frac{2 F^{\prime} F^{\prime \prime} A-\left(F^{\prime}\right)^{2} A^{\prime}}{A^{2}}=2 F F^{\prime} \text { and } \frac{2 G^{\prime} G^{\prime \prime} A-\left(G^{\prime}\right)^{2} A^{\prime}}{A^{2}}=2 G G^{\prime} .
$$

As $F^{\prime} \neq 0, G^{\prime} \neq 0$, we have

$$
\frac{2 F^{\prime \prime} A-F^{\prime} A^{\prime}}{A^{2}}=2 F \quad \text { and } \quad \frac{2 G^{\prime \prime} A-G^{\prime} A^{\prime}}{A^{2}}=2 G .
$$

We add them side by side to obtain

$$
\frac{2\left(F^{\prime \prime}+G^{\prime \prime}\right) A-\left(F^{\prime}+G^{\prime}\right) A^{\prime}}{A^{2}}=2(F+G)
$$

We multiply (4) by $F^{\prime}+G^{\prime}$ to have

$$
\left(\frac{\left(F^{\prime}+G^{\prime}\right)^{2}}{A}\right)^{\prime}=\left\{(F+G)^{2}\right\}^{\prime}
$$

and integrate (5) to obtain

$$
\frac{\left(F^{\prime}+G^{\prime}\right)^{2}}{A}=(F+G)^{2}+c^{\prime}
$$

where $c^{\prime}$ is a constant. From (3) and (6) we have

$$
\frac{F^{\prime} G^{\prime}}{A}=F G+c
$$

where $c=c^{\prime} / 2+1$. Eliminating $A, F^{\prime}$ and $G^{\prime}$ from (3) and (7) we have

$$
\left(F^{2}-1\right)\left(G^{2}-1\right)=(F G+c)^{2}
$$

that is to say,

$$
F^{2}+2 c F G+G^{2}=1-c^{2}
$$

(a) When $c=1, \quad G=-F$; namely, $g=1-f$ and we have $T(r, g)=$ $T(r, f)+O(1)$.

(b) When $c=-1, G=F$; namely, $g=f$ and we have $T(r, g)=T(r, f)$.

(c) When $c^{2} \neq 1$, we divide (8) by $G^{2}$ to obtain

and

$$
\left(\frac{F}{G}\right)^{2}+2 c \frac{F}{G}+1=\frac{1-c^{2}}{G^{2}}
$$

$$
2 T(r, G)=2 T\left(r, \frac{1}{G}\right)+O(1)=2 T\left(r, \frac{F}{G}\right)+O(1)
$$


By symmetry we also have

$$
2 T(r, F)=2 T\left(r, \frac{1}{F}\right)+O(1)=2 T\left(r, \frac{G}{F}\right)+O(1) .
$$

Since $T(r, F / G)=T(r, G / F)+O(1)$, we have from (9) and (10)

$$
T(r, g)=T(r, G)+O(1)=T(r, F)+O(1)=T(r, f)+O(1) .
$$

(II) Let $f(z)$ be a transcendental meromorphic function in the complex plane and let $a, b$ be distinct elements in $\boldsymbol{S}(f)$ such that $0,1, a$ and $b$ are different from each other and such that at least one of $a$ and $b$ is not constant and put

$$
\Delta_{f}=\left|\begin{array}{ccc}
f(f-1) & \left(f^{\prime}\right)^{2} & f f^{\prime} \\
a(a-1) & \left(a^{\prime}\right)^{2} & a a^{\prime} \\
b(b-1) & \left(b^{\prime}\right)^{2} & b b^{\prime}
\end{array}\right| .
$$

Then, we have the following

LEMMA 5. If $\Delta_{f} \neq 0$, we have the inequality

$$
2 T(r, f)<N_{1)}\left(r, \frac{1}{f}\right)+\bar{N}\left(r, \frac{1}{f-1}\right)+\bar{N}\left(r, \frac{1}{f-a}\right)+\bar{N}\left(r, \frac{1}{f-b}\right)+2 \bar{N}(r, f)+S(r, f) \text {. }
$$

Proof of Lemma 5. As in the proof of Lemma 2 in [8], we put

$$
\delta(z)=\frac{1}{3} \min \{1,|a(z)|,|b(z)|,|a(z)-1|,|b(z)-1|,|a(z)-b(z)|\}
$$

and

$$
\theta_{j}(r)=\left\{0 \leq \theta<2 \pi:\left|f\left(r e^{i \theta}\right)-d_{j}\left(r e^{i \theta}\right)\right| \leq \delta\left(r e^{i \theta}\right)\right\},
$$

where $d_{1}=0, d_{2}=1, d_{3}=a$ and $d_{4}=b$. Then,

$$
\frac{1}{2 \pi} \int_{0}^{2 \pi} \log \frac{1}{\delta\left(r e^{i \theta}\right)} d \theta=S(r, f)
$$

(see [8], p. 828).

We note that

$$
\begin{aligned}
f f^{\prime}-a a^{\prime} & =(f-a)\left(f^{\prime}-a^{\prime}\right)+a^{\prime}(f-a)+a\left(f^{\prime}-a^{\prime}\right), \\
f^{2}-f-\left(a^{2}-a\right) & =(f-a)(f+a-1), \\
\left(f^{\prime}\right)^{2}-\left(a^{\prime}\right)^{2} & =\left(f^{\prime}-a^{\prime}\right)\left(f^{\prime}+a^{\prime}\right), \\
f & =f-a+a, \\
f^{\prime} & =f^{\prime}-a^{\prime}+a^{\prime}=\frac{(f-a)\left(f^{\prime}-a^{\prime}\right)}{f-a}+a^{\prime} .
\end{aligned}
$$


When $\theta \in \theta_{3}(r)$

$$
\left|f\left(r e^{i \theta}\right)-a\left(r e^{i \theta}\right)\right| \leq \delta\left(r e^{i \theta}\right) \leq\left|a\left(r e^{i \theta}\right)\right|
$$

and we have from (12) through (16) that for a positive constant $K$

$$
\begin{aligned}
\frac{1}{2 \pi} \int_{\theta_{3}(r)} \log ^{+}\left|\frac{\Delta_{f}\left(r e^{i \theta}\right)}{f\left(r e^{i \theta}\right)-a\left(r e^{i \theta}\right)}\right| d \theta \leq & K\left\{m\left(r, \frac{f^{\prime}-a^{\prime}}{f-a}\right)+m(r, a)\right. \\
& \left.+m(r, b)+m\left(r, a^{\prime}\right)+m\left(r, b^{\prime}\right)+1\right\} \\
= & S(r, f)
\end{aligned}
$$

and from (17) and (11) that

$$
\begin{aligned}
m\left(r, \frac{1}{f-a}\right) \leq & \frac{1}{2 \pi} \int_{\theta_{3}(r)} \log ^{+}\left|\frac{\Delta_{f}\left(r e^{i \theta}\right)}{f\left(r e^{i \theta}\right)-a\left(r e^{i \theta}\right)}\right| d \theta \\
& +\frac{1}{2 \pi} \int_{\theta_{3}(r)} \log ^{+}\left|\frac{1}{\Delta_{f}\left(r e^{i \theta}\right)}\right| d \theta \\
& +\frac{1}{2 \pi} \int_{[0,2 \pi]-\theta_{3}(r)} \log \frac{1}{\delta\left(r e^{i \theta}\right)} d \theta+O(1) \\
\leq & \frac{1}{2 \pi} \int_{\theta_{3}(r)} \log ^{+}\left|\frac{1}{\Delta_{f}\left(r e^{i \theta}\right)}\right| d \theta+S(r, f) .
\end{aligned}
$$

As in the case of (18), we have the following inequalities:

$$
\begin{aligned}
m\left(r, \frac{1}{f-b}\right) & \leq \frac{1}{2 \pi} \int_{\theta_{4}(r)} \log ^{+}\left|\frac{1}{\Delta_{f}\left(r e^{i \theta}\right)}\right| d \theta+S(r, f) ; \\
m\left(r, \frac{1}{f}\right) & \leq \frac{1}{2 \pi} \int_{\theta_{1}(r)} \log ^{+}\left|\frac{1}{\Delta_{f}\left(r e^{i \theta}\right)}\right| d \theta+S(r, f) ; \\
m\left(r, \frac{1}{f-1}\right) & \leq \frac{1}{2 \pi} \int_{\theta_{2}(r)} \log ^{+}\left|\frac{1}{\Delta_{f}\left(r e^{i \theta}\right)}\right| d \theta+S(r, f) .
\end{aligned}
$$

According to the definition of $\theta_{j}(r)$, the sets

$$
\theta_{i}(r) \cap \theta_{j}(r) \quad(1 \leq i \neq j \leq 4)
$$

consist of at most a finite number of elements, so that we have the inequality

$$
m\left(r, \frac{1}{f}\right)+m\left(r, \frac{1}{f-1}\right)+m\left(r, \frac{1}{f-a}\right)+m\left(r, \frac{1}{f-b}\right) \leq m\left(r, \frac{1}{\Delta_{f}}\right)+S(r, f)
$$


from (18), (19), (20) and (21) and by adding the quantity

$$
N\left(r, \frac{1}{f}\right)+N\left(r, \frac{1}{f-1}\right)+N\left(r, \frac{1}{f-a}\right)+N\left(r, \frac{1}{f-b}\right)
$$

to both sides of this inequality we have

$$
\begin{aligned}
4 T(r, f) \leq & N\left(r, \frac{1}{f}\right)+N\left(r, \frac{1}{f-1}\right)+N\left(r, \frac{1}{f-a}\right) \\
& +N\left(r, \frac{1}{f-b}\right)+m\left(r, \frac{1}{\Delta_{f}}\right)+S(r, f) .
\end{aligned}
$$

By the first fundamental theorem of Nevanlinna

$$
m\left(r, \frac{1}{\Delta_{f}}\right)=T\left(r, \Delta_{f}\right)-N\left(r, \frac{1}{\Delta_{f}}\right)+O(1)
$$

and by the definition of $\Delta_{f}$ we have

$$
\begin{aligned}
& m\left(r, \Delta_{f}\right) \leq 2 m(r, f)+S(r, f), \\
& N\left(r, \Delta_{f}\right) \leq 2 N(r, f)+2 \bar{N}(r, f)+S(r, f)
\end{aligned}
$$

and so

$$
T\left(r, \Delta_{f}\right) \leq 2 T(r, f)+2 \bar{N}(r, f)+S(r, f) .
$$

Further, as

$$
\begin{aligned}
n\left(r, \frac{1}{\Delta_{f}}\right) \geq & n_{(2}\left(r, \frac{1}{f}\right)+\left\{n\left(r, \frac{1}{f-1}\right)-\bar{n}\left(r, \frac{1}{f-1}\right)\right\} \\
+ & \left\{n\left(r, \frac{1}{f-a}\right)-\bar{n}\left(r, \frac{1}{f-a}\right)\right\}+\left\{n\left(r, \frac{1}{f-b}\right)-\bar{n}\left(r, \frac{1}{f-b}\right)\right\} \\
& -O\{n(r, a)+n(r, b)\},
\end{aligned}
$$

we have

$$
\begin{aligned}
N\left(r, \frac{1}{\Delta_{f}}\right)+O(\log r) \geq & N_{(2}\left(r, \frac{1}{f}\right)+\left\{N\left(r, \frac{1}{f-1}\right)-\bar{N}\left(r, \frac{1}{f-1}\right)\right\} \\
& +\left\{N\left(r, \frac{1}{f-a}\right)-\bar{N}\left(r, \frac{1}{f-a}\right)\right\} \\
& +\left\{N\left(r, \frac{1}{f-b}\right)-\bar{N}\left(r, \frac{1}{f-b}\right)\right\}+S(r, f) .
\end{aligned}
$$


From (22), (23), (24) and (25), we have the inequality

$$
\begin{aligned}
2 T(r, f)< & N_{1)}\left(r, \frac{1}{f}\right)+\bar{N}\left(r, \frac{1}{f-1}\right)+\bar{N}\left(r, \frac{1}{f-a}\right) \\
& +\bar{N}\left(r, \frac{1}{f-b}\right)+2 \bar{N}(r, f)+S(r, f) .
\end{aligned}
$$

LEMMA 6. If $\Delta_{f}=0$, then following relations hold:

(a) $N_{1)}\left(r, \frac{1}{f}\right)=S(r, f)$

(b) $\bar{N}_{(2}\left(r, \frac{1}{f-1}\right)=S(r, f)$,

(c) $\bar{N}(r, f)=S(r, f)$.

Proof of Lemma 6. Since $\Delta_{f}=0$,

$$
\frac{\Delta_{f}}{f(f-1) a(a-1) b(b-1)}=\left|\begin{array}{ccc}
1 & \frac{\left(f^{\prime}\right)^{2}}{f(f-1)} & \frac{f^{\prime}}{f-1} \\
1 & \frac{\left(a^{\prime}\right)^{2}}{a(a-1)} & \frac{a^{\prime}}{a-1} \\
1 & \frac{\left(b^{\prime}\right)^{2}}{b(b-1)} & \frac{b^{\prime}}{b-1}
\end{array}\right|=0
$$

and we have

$$
\left(\frac{\left(f^{\prime}\right)^{2}}{f(f-1)}-\frac{\left(a^{\prime}\right)^{2}}{a(a-1)}\right)\left(\frac{b^{\prime}}{b-1}-\frac{a^{\prime}}{a-1}\right)=\left(\frac{f^{\prime}}{f-1}-\frac{a^{\prime}}{a-1}\right)\left(\frac{\left(b^{\prime}\right)^{2}}{b(b-1)}-\frac{\left(a^{\prime}\right)^{2}}{a(a-1)}\right) \text {. }
$$

Since $a \in \boldsymbol{S}(f)$, note that by Lemma 4

$$
\frac{\left(f^{\prime}\right)^{2}}{f(f-1)} \neq \frac{\left(a^{\prime}\right)^{2}}{a(a-1)}
$$

and that

$$
\frac{f^{\prime}}{f-1} \neq \frac{a^{\prime}}{a-1}
$$

Then,

$$
\frac{b^{\prime}}{b-1} \neq \frac{a^{\prime}}{a-1} \quad \text { and } \quad \frac{\left(b^{\prime}\right)^{2}}{b(b-1)} \neq \frac{\left(a^{\prime}\right)^{2}}{a(a-1)}
$$

In fact, if one of them does not hold, then the other also does not hold by (27), (28) and (29) and we have $a=b$, which is a contradiction. 

and satisfies

(a) If $N_{1)}(r, 1 / f) \neq S(r, f)$, there is a point $z_{0}$ which is a simple zero of $f$

$$
\begin{gathered}
a\left(z_{0}\right) \neq 0,1, \infty ; b\left(z_{0}\right) \neq 0,1, \infty ;\left(\frac{b^{\prime}}{b-1}-\frac{a^{\prime}}{a-1}\right)\left(z_{0}\right) \neq 0 ; \\
\left(\frac{\left(b^{\prime}\right)^{2}}{b(b-1)}-\frac{\left(a^{\prime}\right)^{2}}{a(a-1)}\right)\left(z_{0}\right) \neq 0 .
\end{gathered}
$$

The left-hand side of (27) has a simple pole at $z_{0}$ and the right-hand side of (27) is regular at $z_{0}$. This is a contradiction. We have (a) of this lemma.

(b) If $\bar{N}_{(2}(r, 1 /(f-1)) \neq S(r, f)$, there is a point $z_{0}$ which is a zero of $f-1$ of order at least 2 and satisfies (30). Then the left-hand side of (27) is regular at $z_{0}$, but the right-hand side of (32) has a pole of order 1. This is a contradiction. We have (b) of this lemma.

(c) If $\bar{N}(r, f) \neq S(r, f)$, there is a point $z_{0}$ which is a pole of $f$ and satisfies (30). The left-hand side of (27) has a double pole at $z_{0}$ and the right-hand side of (27) has a simple pole at $z_{0}$. This is a contradiction. We have (c) of this lemma.

(III) Further we shall give a lemma for later use.

LEMMA 7. Let $f$ and $g$ be two transcendental meromorphic functions in the complex plane satisfying

$$
\bar{N}(r, f)=S(r, f) \quad \text { and } \quad \bar{N}(r, g)=S(r, g) .
$$

We suppose that there are four distinct meromorphic functions $a_{1}, \ldots, a_{4}$ in $\boldsymbol{S}(f) \cap$ $\boldsymbol{S}(g)$ satisfying the condition

$$
E\left(f=a_{j}\right)=E\left(g=a_{j}\right) \quad(j=1,2,3,4) .
$$

Then the following relations hold:

(a) $T(r, g)=T(r, f)+S(r, f)$ and $S(r, g)=S(r, f)$.

(b) If $f \neq g$, then

$$
2 T(r, f)=\sum_{j=1}^{4} \bar{N}\left(r, \frac{1}{f-a_{j}}\right)+S(r, f) .
$$

Proof of Lemma 7. (a) is trivial when $f=g$. We prove (a) and (b) when $f \neq g$.

(a) By Lemma 2 for $a_{5}=\infty$, we have

$$
\begin{aligned}
2 T(r, f) & \leq \sum_{j=1}^{4} \bar{N}\left(r, \frac{1}{f-a_{j}}\right)+S(r, f) \\
& \leq \bar{N}\left(r, \frac{1}{f-g}\right)+S(r, f) \\
& \leq T(r, f)+T(r, g)+S(r, f)
\end{aligned}
$$


and so

$$
T(r, f) \leq T(r, g)+S(r, f) .
$$

By symmetry, we also have from (32) that

$$
T(r, g) \leq T(r, f)+S(r, g) .
$$

From (32) and (33) we have (a) in this case.

(b) By (a) and (31) we have (b) immediately.

\section{Theorem}

In this section we shall prove several unicity theorems for transcendental meromorphic functions in the complex plane sharing four distinct small meromorphic functions and satisfying some conditions on the counting functions of pole.

THEOREM 1. Let $f$ and $g$ be transcendental meromorphic functions in the complex plane. Suppose that there are four distinct elements $a_{1}, a_{2}, a_{3}, a_{4} \in C \cup$ $\{\infty\}$ satisfying

$$
E\left(f=a_{j}\right)=E\left(g=a_{j}\right) \quad(j=1,2,3,4) .
$$

If there exists an element $a_{5}$ in $C \cup\{\infty\}-\left\{a_{1}, a_{2}, a_{3}, a_{4}\right\}$ satisfying

$$
\bar{N}\left(r, \frac{1}{f-a_{5}}\right) \leq u T(r, f)+S(r, f)
$$

or

$$
\bar{N}\left(r, \frac{1}{g-a_{5}}\right) \leq u T(r, g)+S(r, g)
$$

for some $u \in[0,1)$. Then, $f=g$.

Proof of Theorem 1. We may suppose without loss of generality that $a_{1}, \ldots, a_{4}$ are in $C$ and $a_{5}=\infty$ by considering a suitable linear transformation if necessary.

Now, suppose that (35) holds and that $f \neq g$. By Lemma 1 (a) for $q=5$, (34) and (35) we obtain

$$
\begin{aligned}
3 T(r, f) & \leq \sum_{j=1}^{4} \bar{N}\left(r, \frac{1}{f-a_{j}}\right)+\bar{N}\left(r, \frac{1}{f-a_{5}}\right)+S(r, f) \\
& \leq \bar{N}\left(r, \frac{1}{f-g}\right)+u T(r, f)+S(r, f) \\
& \leq T(r, f)+T(r, g)+u T(r, f)+S(r, f)
\end{aligned}
$$


which reduces to

$$
(2-u) T(r, f) \leq T(r, g)+S(r, f)
$$

and similarly we obtain

$$
T(r, g) \leq T(r, f)+S(r, g) .
$$

From (37) and (38) we obtain that $S(r, f)=S(r, g)$ as $0 \leq u<1$ and that

$$
(2-u) T(r, f) \leq T(r, f)+S(r, f),
$$

which arrives at a contradiction

$$
(1-u) T(r, f) \leq S(r, f)
$$

since $1-u>0$ in this case. This shows that $f=g$ must hold.

Similarly we obtain the same conclusion when (36) holds.

THEOREM 2. Let $f$ and $g$ be transcendental meromorphic functions in the complex plane. Suppose that there are four distinct elements $a_{1}, a_{2}, a_{3}, a_{4} \in \boldsymbol{S}(f) \cap$ $\boldsymbol{S}(g)$ satisfying

$$
E\left(f=a_{j}\right)=E\left(g=a_{j}\right) \quad(j=1,2,3,4)
$$

and suppose that there exist $a_{j}, a_{k} \in\left\{a_{1}, a_{2}, a_{3}, a_{4}\right\}(j \neq k)$ such that

$$
a=\frac{c-a_{j}}{a_{k}-a_{j}} \quad \text { and } \quad b=\frac{d-a_{j}}{a_{k}-a_{j}}
$$

are constants, where $\{c, d\}=\left\{a_{1}, a_{2}, a_{3}, a_{4}\right\}-\left\{a_{j}, a_{k}\right\}$.

If either

(i) $\bar{N}(r, f) \leq u T(r, f)+S(r, f) \quad$ or $\quad$ (ii) $\bar{N}(r, g) \leq u T(r, g)+S(r, g)$

holds for some constant $u \in[0,1)$, then $f=g$.

Proof of Theorem 2. Put $F=\left(f-a_{j}\right) /\left(a_{k}-a_{j}\right)$ and $G=\left(g-a_{j}\right) /\left(a_{k}-a_{j}\right)$, then $0,1, a, b$ are distinct and we obtain the relations

$$
\begin{gathered}
T(r, F)=T(r, f)+S(r, f), \quad \bar{N}\left(r, \frac{1}{F}\right)=\bar{N}\left(r, \frac{1}{f-a_{j}}\right)+S(r, f), \\
\bar{N}\left(r, \frac{1}{F-1}\right)=\bar{N}\left(r, \frac{1}{f-a_{k}}\right)+S(r, f), \\
\bar{N}\left(r, \frac{1}{F-a}\right)=\bar{N}\left(r, \frac{1}{f-c}\right)+S(r, f), \\
\bar{N}\left(r, \frac{1}{F-b}\right)=\bar{N}\left(r, \frac{1}{f-d}\right)+S(r, f), \quad \bar{N}(r, F)=\bar{N}(r, f)+S(r, f)
\end{gathered}
$$


and

$$
S(r, F)=S(r, f)
$$

The similar relations for $g$ and $G$ hold.

Suppose now that (i) holds and that $f \neq g$. Then, by Lemma $1(\mathrm{a})$ for $q=5$ and by using the above relations and (39) we have

$$
\begin{aligned}
3 T(r, f)+S(r, f)= & 3 T(r, F) \\
\leq & \bar{N}\left(r, \frac{1}{F}\right)+\bar{N}\left(r, \frac{1}{F-1}\right)+\bar{N}\left(r, \frac{1}{F-a}\right)+\bar{N}\left(r, \frac{1}{F-b}\right) \\
& +\bar{N}(r, F)+S(r, F) \\
= & \sum_{j=1}^{4} \bar{N}\left(r, \frac{1}{f-a_{j}}\right)+\bar{N}(r, f)+S(r, f) \\
\leq & T(r, f)+T(r, g)+\bar{N}(r, f)+S(r, f),
\end{aligned}
$$

which reduces to

$$
(2-u) T(r, f) \leq T(r, g)+S(r, f)
$$

by (i). Similarly we have

$$
T(r, g) \leq T(r, f)+S(r, g) .
$$

From (40) and (41) we obtain that $S(r, f)=S(r, g)$ since $u \in[0,1)$ and that

$$
(2-u) T(r, f) \leq T(r, f)+S(r, f),
$$

which arrives at a contradiction

$$
(1-u) T(r, f)=S(r, f)
$$

since $1-u>0$. This shows that $f=g$ must hold in this case.

Similarly we obtain the same conclusion when (ii) holds.

THEOREM 3. Let $f$ and $g$ be transcendental meromorphic functions in the complex plane. Suppose that there are four distinct elements $a_{1}, a_{2}, a_{3}, a_{4} \in \boldsymbol{S}(f) \cap$ $\boldsymbol{S}(g)$ satisfying

$$
E\left(f=a_{j}\right)=E\left(g=a_{j}\right) \quad(j=1,2,3,4)
$$

and suppose that for any $j, k(1 \leq j \neq k \leq 4)$, at least one of

$$
a=\frac{c-a_{j}}{a_{k}-a_{j}} \quad \text { and } \quad b=\frac{d-a_{j}}{a_{k}-a_{j}}
$$

is not constant, where $\{c, d\}=\left\{a_{1}, a_{2}, a_{3}, a_{4}\right\}-\left\{a_{j}, a_{k}\right\}$. (d):

If $\bar{N}(r, f)$ and $\bar{N}(r, g)$ satisfy one of the following conditions (a), (b), (c) and 
(a) $\bar{N}(r, f)=S(r, f)$ and $\bar{N}(r, g)=S(r, g)$;

(b) $\bar{N}(r, g)=S(r, g), \bar{N}(r, f) \neq S(r, f)$ and $\bar{N}(r, f) \leq u T(r, f)+S(r, f)$

for some $u \in(0,1 / 19)$;

(c) $\bar{N}(r, f)=S(r, f), \bar{N}(r, g) \neq S(r, g)$ and $\bar{N}(r, g) \leq v T(r, g)+S(r, g)$

for some $v \in(0,1 / 19)$;

(d) $\bar{N}(r, f) \neq S(r, f), \bar{N}(r, g) \neq S(r, g)$ and

$$
\bar{N}(r, f) \leq u T(r, f)+S(r, f), \quad \bar{N}(r, g) \leq v T(r, g)+S(r, g)
$$

for some $u, v \in(0,1)$ satisfying either

(i) $0<u<1 / 19$ and $0<v<(2-19 u) /(20-19 u)$ or

(ii) $0<v<1 / 19$ and $0<u<(2-19 v) /(20-19 v)$, then $f=g$.

Proof of Theorem 3. CASE 1. The case when (a) is satisfied. Suppose that $f \neq g$.

SubCASE 1-1. The case when for at least one $j(1 \leq j \leq 4)$

$$
\bar{N}\left(r, \frac{1}{f-a_{j}}\right)=S(r, f) .
$$

Suppose without loss of generality that (43) holds for $j=1$. Then by Lemma 1(c) and Lemma 7(b) we have

$$
\begin{aligned}
3 T(r, f) & \leq \sum_{j=1}^{4} \bar{N}\left(r, \frac{1}{f-a_{j}}\right)+2 \bar{N}\left(r, \frac{1}{f-a_{1}}\right)+S(r, f) \\
& =2 T(r, f)+S(r, f),
\end{aligned}
$$

which reduces to $T(r, f)=S(r, f)$. This is a contradiction.

SubCASE 1-2. The case when for any $j(1 \leq j \leq 4)$

$$
\bar{N}\left(r, \frac{1}{f-a_{j}}\right) \neq S(r, f) .
$$

We shall arrive at a contradiction by five steps.

STEP 1. For each $j$, either

(i) $\bar{N}_{(2}\left(r, \frac{1}{f-a_{j}}\right)=S(r, f)$ or $\left(\right.$ ii) $N_{1)}\left(r, \frac{1}{f-a_{j}}\right)=S(r, f)$.

We shall prove Step 1 for $j=1$. We put

$$
F=\frac{f-a_{1}}{a_{2}-a_{1}}, \quad a=\frac{a_{3}-a_{1}}{a_{2}-a_{1}} \quad \text { and } \quad b=\frac{a_{4}-a_{1}}{a_{2}-a_{1}} \text {. }
$$


Then, $0,1, a$ and $b$ are different from each other; $a, b \in \boldsymbol{S}(f) \cap \boldsymbol{S}(g)$ and at least one of $a, b$ is not constant by the assumption; $F$ is transcendental and we have

$$
\begin{cases}T(r, F)=T(r, f)+S(r, f), & S(r, F)=S(r, f) \\ \bar{N}\left(r, \frac{1}{F}\right)=\bar{N}\left(r, \frac{1}{f-a_{1}}\right)+S(r, f), & N_{1)}\left(r, \frac{1}{F}\right)=N_{1)}\left(r, \frac{1}{f-a_{1}}\right)+S(r, f) \\ \bar{N}\left(r, \frac{1}{F-1}\right)=\bar{N}\left(r, \frac{1}{f-a_{2}}\right)+S(r, f), & \bar{N}_{(2}\left(r, \frac{1}{F-1}\right)=\bar{N}_{(2}\left(r, \frac{1}{f-a_{2}}\right)+S(r, f) \\ \bar{N}\left(r, \frac{1}{F-a}\right)=\bar{N}\left(r, \frac{1}{f-a_{3}}\right)+S(r, f), & \bar{N}\left(r, \frac{1}{F-b}\right)=\bar{N}\left(r, \frac{1}{f-a_{4}}\right)+S(r, f)\end{cases}
$$

and

$$
\bar{N}(r, F)=S(r, F) .
$$

We shall show that if $\Delta_{F} \neq 0$ then (i) holds, and show that if $\Delta_{F}=0$ then (ii) holds. First we treat the case when $\Delta_{F} \neq 0$. By applying Lemma 5 to $F$ we obtain from (46) that

$$
\begin{aligned}
2 T(r, f) & +S(r, f)=2 T(r, F) \\
\leq & N_{1)}\left(r, \frac{1}{F}\right)+\bar{N}\left(r, \frac{1}{F-1}\right)+\bar{N}\left(r, \frac{1}{F-a}\right)+\bar{N}\left(r, \frac{1}{F-b}\right)+S(r, F) \\
& =N_{1)}\left(r, \frac{1}{f-a_{1}}\right)+\sum_{j=2}^{4} \bar{N}\left(r, \frac{1}{f-a_{j}}\right)+S(r, f),
\end{aligned}
$$

so that we have

$$
\begin{aligned}
2 T(r, f)+\bar{N}_{(2}\left(r, \frac{1}{f-a_{1}}\right) & \leq \sum_{j=1}^{4} \bar{N}\left(r, \frac{1}{f-a_{j}}\right)+S(r, f) \\
& =2 T(r, f)+S(r, f)
\end{aligned}
$$

by Lemma $7($ b) as $f \neq g$. This implies that

$$
\bar{N}_{(2}\left(r, \frac{1}{f-a_{1}}\right)=S(r, f) .
$$

Secondly, we treat the case when $\Delta_{F}=0$. By applying Lemma 6(a) to $F$ we obtain from (45) that

namely,

$$
N_{1)}\left(r, \frac{1}{f-a_{1}}\right)+S(r, f)=N_{1)}\left(r, \frac{1}{F}\right)=S(r, F)=S(r, f)
$$

$$
N_{1)}\left(r, \frac{1}{f-a_{1}}\right)=S(r, f)
$$


We can prove Step 1 for $j=2,3,4$ in the same way.

STEP 2. There is at most one $j(1 \leq j \leq 4)$ for which the relation

$$
N_{1)}\left(r, \frac{1}{f-a_{j}}\right)=S(r, f)
$$

holds.

In fact, suppose to the contrary that there are two $j$ 's for which (47) holds. For example we suppose that

$$
N_{1)}\left(r, \frac{1}{f-a_{1}}\right)=S(r, f) \quad \text { and } \quad N_{1)}\left(r, \frac{1}{f-a_{2}}\right)=S(r, f) .
$$

Let $F, a$ and $b$ be as in Step 1. Then we assert that $\Delta_{F}=0$ in this case. In fact, if $\Delta_{F} \neq 0$, then by Step $1(i)$ we have that

$$
\bar{N}_{(2}\left(r, \frac{1}{f-a_{1}}\right)=S(r, f) \text {. }
$$

From (48) and (49) we have that

$$
\bar{N}\left(r, \frac{1}{f-a_{1}}\right)=N_{1)}\left(r, \frac{1}{f-a_{1}}\right)+\bar{N}_{(2}\left(r, \frac{1}{f-a_{1}}\right)=S(r, f),
$$

which contradicts with (44). This shows that $\Delta_{F}=0$. Then, by applying Lemma $6($ b) to $F$ we have from (45) that

$$
\bar{N}_{(2}\left(r, \frac{1}{f-a_{2}}\right)+S(r, f)=\bar{N}_{(2}\left(r, \frac{1}{F-1}\right)=S(r, F)=S(r, f) ;
$$

that is,

$$
\bar{N}_{(2}\left(r, \frac{1}{f-a_{2}}\right)=S(r, f) .
$$

From (48) and (50) we have that

$$
\bar{N}\left(r, \frac{1}{f-a_{2}}\right)=N_{1)}\left(r, \frac{1}{f-a_{2}}\right)+\bar{N}_{(2}\left(r, \frac{1}{f-a_{2}}\right)=S(r, f),
$$

which contradicts with (44). This completes the proof of Step 2.

\section{STEP 3. Either}

(i) $\bar{N}_{(2}\left(r, 1 /\left(f-a_{j}\right)\right)=S(r, f)(j=1,2,3,4)$ or

(ii) $N_{1)}\left(r, 1 /\left(f-a_{j}\right)\right)=S(r, f)$ for one $j(1 \leq j \leq 4)$ and $\bar{N}_{(2}\left(r, 1 /\left(f-a_{k}\right)\right)=$ $S(r, f)$ for any $k(1 \leq k \leq 4, k \neq j)$. 
This is a direct consequence of Step 1 and Step 2.

STEP 4. In case of Step 3(i), for any integer $m$ the inequality

$$
\begin{aligned}
N_{m}\left(r, \frac{1}{f-a_{j}}\right) & \leq \bar{N}\left(r, \frac{1}{f-a_{j}}\right)+(m-1) \bar{N}_{(2}\left(r, \frac{1}{f-a_{j}}\right) \\
& =\bar{N}\left(r, \frac{1}{f-a_{j}}\right)+S(r, f)
\end{aligned}
$$

holds for $j=1,2,3,4$ and so we have from Lemma 3 and Lemma 7 (b) that

$$
3 T(r, f) \leq \sum_{j=1}^{4} \bar{N}\left(r, \frac{1}{f-a_{j}}\right)+S(r, f)=2 T(r, f)+S(r, f),
$$

which reduces to $T(r, f)=S(r, f)$. This is a contradiction.

STEP 5. In case of Step 3(ii), we suppose without loss of generality that

$$
N_{1)}\left(r, \frac{1}{f-a_{1}}\right)=S(r, f) \quad \text { and } \quad \bar{N}_{(2}\left(r, \frac{1}{f-a_{j}}\right)=S(r, f) \quad(j=2,3,4) \text {. }
$$

As the inequality (51) holds for $j=2,3,4$ in this case, we have by Lemma 3 for $q=4,1 \leq m \leq 4$ and Lemma $7($ b) that

$$
\begin{aligned}
3 T(r, f) & \leq \sum_{j=1}^{4} N_{m}\left(r, \frac{1}{f-a_{j}}\right)+S(r, f) \\
& \leq N_{m}\left(r, \frac{1}{f-a_{1}}\right)+\sum_{j=2}^{4} \bar{N}\left(r, \frac{1}{f-a_{j}}\right)+S(r, f) \\
& =N_{m}\left(r, \frac{1}{f-a_{1}}\right)-\bar{N}\left(r, \frac{1}{f-a_{1}}\right)+2 T(r, f)+S(r, f)
\end{aligned}
$$

since $f \neq g$, and so we obtain the inequality

$$
T(r, f)+\bar{N}\left(r, \frac{1}{f-a_{1}}\right) \leq N_{m}\left(r, \frac{1}{f-a_{1}}\right)+S(r, f) \leq T(r, f)+S(r, f)
$$

since the inequality $N_{m}\left(r, 1 /\left(f-a_{1}\right)\right) \leq T(r, f)+S(r, f)$ holds in general. This implies that

$$
\bar{N}\left(r, \frac{1}{f-a_{1}}\right)=S(r, f)
$$

which contradicts with (44).

From Subcases 1 and $2, f$ must be equal to $g$ when (a) is satisfied.

To deal with the cases when $(b),(c)$ or (d) is satisfied, we shall prepare some inequalities. 
Suppose that $f \neq g$. If $(*)$ is satisfied for $(u, v) \in[0,1) \times[0,1)$, then by Lemma 2 for $a_{5}=\infty$ we obtain

$$
\begin{aligned}
2 T(r, f) & \leq \sum_{j=1}^{4} \bar{N}\left(r, \frac{1}{f-a_{j}}\right)+\bar{N}(r, f)+S(r, f) \\
& \leq \bar{N}\left(r, \frac{1}{f-g}\right)+\bar{N}(r, f)+S(r, f) \\
& \leq T(r, f)+T(r, g)+u T(r, f)+S(r, f),
\end{aligned}
$$

which reduces to

$$
(1-u) T(r, f) \leq T(r, g)+S(r, f) .
$$

By symmetry we obtain the inequality

$$
(1-v) T(r, g) \leq T(r, f)+S(r, g) .
$$

Since $0 \leq u<1,0 \leq v<1$, we have from (52) and (53)

$$
S(r, f)=S(r, g) \text {. }
$$

Now suppose in addition that $\bar{N}(r, f) \neq S(r, f)$. We apply Lemma 5 to $\varphi=\left(f-a_{j}\right) /\left(a_{k}-a_{j}\right)(1 \leq j \leq 4,1 \leq k \neq j \leq 4)$ to obtain

$$
\begin{aligned}
2 T(r, f)+\bar{N}_{(2}\left(r, \frac{1}{f-a_{j}}\right) & \leq \sum_{i=1}^{4} \bar{N}\left(r, \frac{1}{f-a_{i}}\right)+2 \bar{N}(r, f)+S(r, f) \\
& \leq \bar{N}\left(r, \frac{1}{f-g}\right)+2 \bar{N}(r, f)+S(r, f) \\
& \leq T(r, f)+T(r, g)+2 \bar{N}(r, f)+S(r, f),
\end{aligned}
$$

since $f \neq g$ and so

$$
\bar{N}_{(2}\left(r, \frac{1}{f-a_{j}}\right) \leq T(r, g)-T(r, f)+2 \bar{N}(r, f)+S(r, f) .
$$

Note that $\Delta_{\varphi} \neq 0$ in this case by Lemma $6(\mathrm{c})$ since

$$
\bar{N}(r, \varphi)=\bar{N}(r, f)+S(r, f) \neq S(r, f)=S(r, \varphi) .
$$

Put $F=\left(f-a_{1}\right) /\left(a_{2}-a_{1}\right)$ and apply Lemma 3 to $F$ and $0,1, a=$ $\left(a_{3}-a_{1}\right) /\left(a_{2}-a_{1}\right), b=\left(a_{4}-a_{1}\right) /\left(a_{2}-a_{1}\right)$. Note that $q=4$ and the number $m$ in Lemma 3 satisfies $1 \leq m \leq 3$ in this case. We then have by (55) 


$$
\begin{aligned}
3 T(r, f)+S(r, f)= & 3 T(r, F) \\
\leq & N_{3}\left(r, \frac{1}{F}\right)+N_{3}\left(r, \frac{1}{F-1}\right)+N_{3}\left(r, \frac{1}{F-a}\right) \\
& +N_{3}\left(r, \frac{1}{F-b}\right)+3 \bar{N}(r, F)+S(r, F) \\
= & \sum_{j=1}^{4} N_{3}\left(r, \frac{1}{f-a_{j}}\right)+3 \bar{N}(r, f)+S(r, f) \\
\leq & \sum_{j=1}^{4} \bar{N}\left(r, \frac{1}{f-a_{j}}\right)+\sum_{J=1}^{4} 2 \bar{N}_{(2}\left(r, \frac{1}{f-a_{j}}\right)+3 \bar{N}(r, f)+S(r, f) \\
\leq & \bar{N}\left(r, \frac{1}{f-g}\right)+8(T(r, g)-T(r, f))+19 \bar{N}(r, f)+S(r, f) \\
\leq & T(r, f)+T(r, g)+8(T(r, g)-T(r, f))+19 \bar{N}(r, f)+S(r, f),
\end{aligned}
$$

which reduces to

$$
10 T(r, f) \leq 9 T(r, g)+19 \bar{N}(r, f)+S(r, f) .
$$

Now we return to the proof.

CASE 2. The case when (b) is satisfied. Suppose that $f \neq g$.

As $\bar{N}(r, g)=S(r, g)$, from (53) for $v=0,(54)$ and (56) we obtain

$$
\begin{aligned}
T(r, f) & \leq 19 \bar{N}(r, f)+S(r, f) \\
& \leq 19 u T(r, f)+S(r, f)
\end{aligned}
$$

which reduces to

$$
(1-19 u) T(r, f) \leq S(r, f),
$$

which is a contradiction since $1-19 u>0$ in this case. This implies that $f$ must be equal to $g$ when (b) is satisfied.

CASE 3. The case when (c) is satisfied. Suppose that $f \neq g$.

As in Case 2, we obtain the inequality

$$
(1-19 v) T(r, g) \leq S(r, g)
$$

which is a contradiction since $1-19 v>0$ in this case. This implies that $f$ must be equal to $g$ when (c) is satisfied.

CASE 4. The case when (d) is satisfied. Suppose that $f \neq g$. 
As $\bar{N}(r, f) \neq S(r, f)$ and $\bar{N}(r, g) \neq S(r, g)$, the inequality (56) holds and the following inequality also holds:

$$
10 T(r, g) \leq 9 T(r, f)+19 \bar{N}(r, g)+S(r, g) .
$$

We add (56) and (57) side by side and by the condition (*) we obtain

$$
(1-19 u) T(r, f)+(1-19 v) T(r, g) \leq S(r, f)+S(r, g) .
$$

SubCASE 4-1. The case when $1-19 v \geq 0$ and $(u, v)$ satisfies (i).

Note that $(2-19 u) /(20-19 u)-1 / 19>0$ in this case. The relation $(58)$ is a contradiction since $1-19 u>0$ in this case.

SubCaSe 4-2. The case when $1-19 v<0$ and $(u, v)$ satisfies (i).

From (58) and (53) we obtain the relation

$$
\{(1-19 u)(1-v)+(1-19 v)\} T(r, f) \leq S(r, f),
$$

which is also a contradiction since $(1-19 u)(1-v)+(1-19 v)>0$ in this case.

SubCASE 4-3. The case when $1-19 u \geq 0$ and $(u, v)$ satisfies (ii).

The relation (58) is a contradiction since $1-19 v>0$ in this case.

SubCASE 4-4. The case when $1-19 u<0$ and $(u, v)$ satisfies (ii).

From (58) and (52) we obtain the relation

$$
\{(1-19 v)(1-u)+(1-19 u)\} T(r, f) \leq S(r, f),
$$

which is also a contradiction since $(1-19 v)(1-u)+(1-19 u)>0$ in this case.

From Subcase 4-1 through Subcase 4-4 we obtain that $f$ must be equal to $g$ when $(\mathrm{d})$ is satisfied.

We complete the proof of Theorem 3 .

COROLlaRY 1. Let $f$ and $g$ be transcendental meromorphic functions in the complex plane. Suppose that there are four distinct elements $a_{1}, a_{2}, a_{3}, a_{4} \in$ $\boldsymbol{S}(f) \cap \boldsymbol{S}(g)$ satisfying

$$
E\left(f=a_{j}\right)=E\left(g=a_{j}\right) \quad(j=1,2,3,4) .
$$

If the following inequalities

$$
\bar{N}(r, f) \leq u T(r, f)+S(r, f) \quad \text { and } \quad \bar{N}(r, g) \leq v T(r, g)+S(r, g)
$$

hold for some constants $u$ and $v$ satisfying

$$
(u, v) \in\left[0, \frac{1}{19}\right) \times\left[0, \frac{1}{19}\right)
$$

then $f=g$.

In fact, if $(u, v)$ satisfies $(59), \bar{N}(r, f)$ and $\bar{N}(r, g)$ satisfy one of the conditions given in Theorem 2 or Theorem 3. 
COROllary 2. Let $f$ and $g$ be transcendental meromorphic functions in $|z|<\infty$. Suppose that there exist five distinct elements $a_{1}, \ldots, a_{5}$ in $\{\boldsymbol{S}(f) \cap \boldsymbol{S}(g)\}$ satisfying the conditions

$$
E\left(f=a_{j}\right)=E\left(g=a_{j}\right) \quad(j=1, \ldots, 5)
$$

and

$$
\bar{N}\left(r, \frac{1}{f-a_{5}}\right) \leq u T(r, f)+S(r, f)
$$

for some $u \in[0,1 / 19)$, then $f=g$.

Proof of Corollary 2. By Lemma 2 we can prove that $T(r, g)=T(r, f)+$ $S(r, f)$ and $S(r, f)=S(r, g)$ in this case as in Lemma 7(a). Then, from the hypotheses of this corollary we have

$$
\bar{N}\left(r, \frac{1}{g-a_{5}}\right) \leq u T(r, g)+S(r, g) .
$$

Then by Corollary 1 we have that $f=g$.

\title{
REFERENCES
}

[1] B.Q. LI, Uniqueness of entıre functions sharıng four small functions, Amer. J. Math., 119 (1997), 841-858.

[2] D. Drasin, Meromorphic functions: progress and problems, Proceedings of the International Congress of Mathematıcians, Zürıch, 1994, Birkhäuser Verlag, Basel, 1995, 828-835.

[3] G. Frank AND G. Weissenborn, On the zeros of linear differential polynomials of meromorphic functions, Complex Variables Theory Appl., 12 (1989), 77-81.

[4] W K. Hayman, Meromorphic Functions, Clarendon Press, Oxford, 1964.

[5] R. Nevanlinna, Le théorème de Picard-Borel et la théorie des fonctions méromorphes, Gauthier-Villars, Paris, 1929.

[6] M. Shirosaki, An extension of unicity theorem for meromorphic functions, Tohoku Math. J., 45 (1993), 491-497

[7] N. Toda, Some generalizations of the unicity theorem of Nevanlinna, Proc. Japan Acad. Ser. A Math. Sc1., 69 (1993), 61-65.

[8] Q.D. ZHANG, A uniqueness theorem for meromorphic functions with respect to slowly growing functions, Acta Math. Sinıca, 36 (1993), 826-833, in Chinese.

\author{
Department of MATHEMatics \\ NiPPON INSTITUTE OF TECHNOLOGY \\ SAITAMA $345-8501$ \\ JAPAN \\ Department of Mathematics \\ NAgoya Institute of TechNology \\ GoKISO, SHOWA-KU \\ NAGOYA 466-8555 \\ JAPAN
}

4-1 Gakuendai Miyashiro, Minamisaitama 